\title{
A Continuous Robust Control Strategy for the Active Aeroelastic Vibration Suppression of Supersonic Lifting Surfaces
}

\author{
K. Zhang* and Z. Wang** \\ Department of EECS at the University of Central Florida
}

\author{
A. Behal*** \\ Department of EECS and NanoScience Technology Center at the University of Central Florida
}

\section{P. Marzocca****}

Mechanical and Aeronautical Engineering at Clarkson University

\begin{abstract}
The model-free control of aeroelastic vibrations of a non-linear 2-D wing-flap system operating in supersonic flight speed regimes is discussed in this paper. A novel continuous robust controller design yields asymptotically stable vibration suppression in both the pitching and plunging degrees of freedom using the flap deflection as a control input. The controller also ensures that all system states remain bounded at all times during closed-loop operation. A Lyapunov method is used to obtain the global asymptotic stability result. The unsteady aerodynamic load is considered by resourcing to the non-linear Piston Theory Aerodynamics (PTA) modified to account for the effect of the flap deflection. Simulation results demonstrate the performance of the robust control strategy in suppressing dynamic aeroelastic instabilities, such as non-linear flutter and limit cycle oscillations.
\end{abstract}

Key words: Nonlinear aeroelastic control, model-free control, robust and neural control, Supersonic aerodynamic

\section{Nomenclature}

$\begin{array}{ll}A, G, G_{d} & \text { State and input matrices } \\ A_{c}, B_{c} & \text { State and input matrices of transformed } \\ & \text { system } \\ A_{c}^{*} & \text { Matrix of the system zero dynamics } \\ a_{\infty}, p_{\infty}, \rho_{\infty} & \text { Sound speed the pressure and air density } \\ & \text { of the undisturbed flow respectively } \\ B & \text { Non-linear restoring moment } \\ C_{0}, C_{1}, C, C_{3} & \text { Constants used in the bounded neural } \\ & \text { network composite weight matrix } \\ \text { e, } \mathbf{r} & \text { Tracking error and filtered tracking error, }\end{array}$

respectively

F, G Positive definite diagonal gain matrixes for update laws of $\hat{\mathbf{W}}$ and $\mathbf{V}$

$g_{1}, g_{2} \quad$ Auxiliary saturation gains

$K_{z}, K_{v}, \Gamma, Z_{b}, K_{d} \quad$ Controller gains

$L_{a}(t), M_{a}(t) \quad$ lifting and aerodynamic moment

$p(y, t) \quad$ Unsteady pressure

$V \quad$ Dimensionless flight speed

$v_{z}(t) \quad$ Downwash velocity normal to the airfoil surface

$W, V \quad$ Ideal neural network interconnection weight matrices
This is an Open Access article distributed under the terms of the Creative Commons Attribution Non-Commercial License (http://creativecommons.org/licenses/by$\mathrm{nc} / 3.0 /$ which permits unrestricted non-commercial use, distribution, and reproduction in any medium, provided the original work is properly cited. (c) * Graduate student, E-mail : kun.zhang@knights.ucf.edu ** Graduate student, E-mail : zwang@knights.ucf.edu *** Corresponding author : Assistant Professor, E-mail : abehal@ucf.edu **** Associate Professor, E-mail : pmarzocc@clarkson.edu 


$\begin{array}{ll}\hat{W}, \hat{V} & \text { Estimated neural network interconnection } \\ w(t) & \text { weight matrices } \\ \mathbf{x}, \mathbf{u} & \text { Transverse deflection } \\ \mathbf{Z}, Z_{\mathrm{B}} & \text { System state and input, respectively } \\ & \text { Ideal neural networks composite weight } \\ \hat{\mathrm{Z}}, \overline{\mathrm{Z}} & \text { matrix and its bound } \\ \bar{\beta} & \text { Estimated neural networks composite } \\ \xi & \text { weight matrix and mismatch } \\ & \text { Auxiliary control input } \\ \gamma & \text { Dimensionless plunging displacement } \\ \tau & \xi=h / b \\ \lambda & \text { Isentropic gas coefficient }(\gamma=1.4 \text { for dry- } \\ \eta(t) & \text { air }) \\ & \text { Dimensionless time } \tau=U_{t} / b \\ \operatorname{tr}\{\cdot\} & \text { Aerodynamic factor } \lambda=M / \sqrt{M^{2}-1} \\ & \text { Vector of system states for analysis of zero } \\ & \text { dynamics } \\ & \text { Trace of a square matrix defined as the } \\ \|\cdot\|_{F} & \text { sum of the elements on the matrix main } \\ <A, B> & \text { diagonal } \\ & \text { Frobenius norm defined as }\|\mathbf{A}\|_{F}= \\ & \sqrt{\operatorname{tr}\left\{A * A^{\mathrm{T}}\right\}} \\ & \text { Inner product of two matrix, defined as } \\ & \operatorname{tr}\{B * A\}\end{array}$

\section{Introduction}

In recent years, aeroelastic control and flutter suppression of flexible wings have been extensively investigated by numerous researchers. There are two basic problems associated with the aeroelastic instability of lifting surfaces - the determination of the flutter boundary and of its character, i.e., the identification of the presence of a stable or unstable Limit Cycle Oscillation (LCO) in the proximity of the flutter boundary. Classical flutter analysis is based on the linearized aeroelastic equations, while LCO analysis requires a nonlinear approach [1]. The goal of the control is to expand the flight envelope above the uncontrolled flutter instability speed without weight penalties and eventually convert the catastrophic nature of flutter, associated with an unstable LCO typical of a subcritical Hopf-bifurcation behavior, into benign flutter, which conversely is associated with a stable LCO typical of a supercritical Hopf-bifurcation. A great deal of research activity devoted to the aeroelastic active control and flutter suppression of flight vehicles has been accomplished, e.g., see [2]. The model nonlinearities can help to stabilize the LCO or be detrimental by destabilizing the LCO [3]. The nonlinearities to be included in the aeroelastic model can be structural [4] (i.e., arising from the kinematic equations); physical [5] (i.e., those involving the constitutive equations); or aerodynamic appearing in the unsteady aerodynamic equations [1][3][6]. This issue is discussed in the context of panel flutter in [1][6][7].

A plethora of techniques is available for dealing with the effect of non-linear structural stiffness in the context of subsonic flow; linear control theory, feedback linearizing techniques, adaptive, and robust control techniques have been employed to account for these nonlinearities, e.g., [8]-[19]. Recently, neural-network-based (i.e., model-free) control approaches have been proposed in [20] and [21] to stabilize a nonlinear aeroelastic wing section. However, there is very little work (e.g., [3],[22]) dealing with the aeroelastic vibration suppression for a supersonic wing section in the presence of both structural and aerodynamic nonlinearities.

Motivated by our previous work in [19][21] and [23]-[25], a novel neural network (NN) based robust controller has been designed to asymptotically stabilize a supersonic aeroelastic system with unstructured nonlinear uncertainties. The nonlinearity of the model depends on the plunging distance and pitching angle. If the nonlinearity is known and could be linearly parameterized, then adaptive control is often considered to be the method of choice. In this paper, we assume unstructured uncertainty in the sense that the structure of the system nonlinearity is considered to be unknown. In contrast to existing neural network-based controllers that only achieve practical stability, the novel continuous control design in this paper is able to achieve asymptotic stability of the origin. A three-layer neural network is implemented to approximate the unknown nonlinearity of the system. While adaptive control relies on linear parameterizability of the system nonlinearity and the determination of a regression matrix, the universal approximation property of the $\mathrm{NN}$ controller enables approximation of the unstructured nonlinear system in a more suitable way. To compensate for the inevitable NN functional approximation error, an integral of a sliding mode term is introduced. Through a Lyapunov analysis, global asymptotic stability can be obtained for the tracking error in the pitching degree of freedom. Then, based on the fact that the system is minimum phase, the asymptotic stability of the plunging degree of freedom is also guaranteed. Simulation results show that this NN-based robust continuous control design can rapidly suppress the flutter and limit cycle oscillations of the aeroelastic system.

The rest of the paper is organized as follows. In Section II, the aeroelastic system dynamics are introduced. In Section III, the control objective is stated explicitly while zero dynamics of the system is analyzed. The open-loop error system is developed in Section IV to facilitate the subsequent control 
design while the closed-loop error system is developed in Section V. In Section VI, Lyapunov-based analysis of the stability of the closed-loop system is presented while the simulation results are shown in Section VII. Appropriate conclusions are drawn in Section VIII.

\section{Model Development}

The aeroelastic governing equations of a supersonic wing section with plunging and twisting degrees-of-freedom (graphically represented in Fig. 1), accounting for flap deflections, and constrained by a linear translational spring and a non-linear torsional spring, are given as follows

$$
\left[\begin{array}{cc}
1 & \chi_{\alpha} \\
\frac{\chi_{\alpha}}{r_{\alpha}} & 1
\end{array}\right]\left[\begin{array}{c}
\ddot{\xi} \\
\ddot{\alpha}
\end{array}\right]+\left[\begin{array}{cc}
2 \zeta_{h}\left(\frac{\bar{\omega}}{V}\right) & 0 \\
0 & 2 \frac{\zeta_{\alpha}}{V}
\end{array}\right]\left[\begin{array}{c}
\dot{\xi} \\
\dot{\alpha}
\end{array}\right]+\left[\begin{array}{cc}
\left(\frac{\bar{\omega}}{V}\right)^{2} & 0 \\
0 & \left(\frac{1}{V^{2}}+\frac{B \alpha^{2}}{V^{2}}\right)
\end{array}\right]\left[\begin{array}{c}
\xi \\
\alpha
\end{array}\right]=\left[\begin{array}{c}
l_{a} \\
m_{a}
\end{array}\right]
$$

The dimensionless plunging distance (positive downward) is expressed as $\xi(\equiv h / b)$, while $\alpha$ is the pitch angle (positive nose up), $\dot{\alpha}$ and $\dot{\xi}$ are derivatives with respect to dimensionless time $\tau=U_{t} / b$, and $\mathrm{V}=U / b \omega_{\alpha}$ is the dimensionless flight speed. The parameter $B$ represents the non-linear restoring moment and is defined as the ratio between the linear and non-linear stiffness coefficients, thus it measures of the degree of nonlinearity of the system; $B>0$ corresponds to hard structural nonlinearities, $B=0$ corresponds to a linear model, while $B$ $<0$ corresponds to soft structural nonlinearities. In addition, $l_{\alpha}$ and $m_{\alpha}$ represent the dimensionless aerodynamic lift and moment with respect to the elastic axis.

In order to account for flap deflections, some modifications need to be made to the non-linear Piston Theory Aerodynamics (PTA) which is used here to produce the aerodynamic loads on the lifting surface. To keep the paper self-contained, a short description of the PTA modified

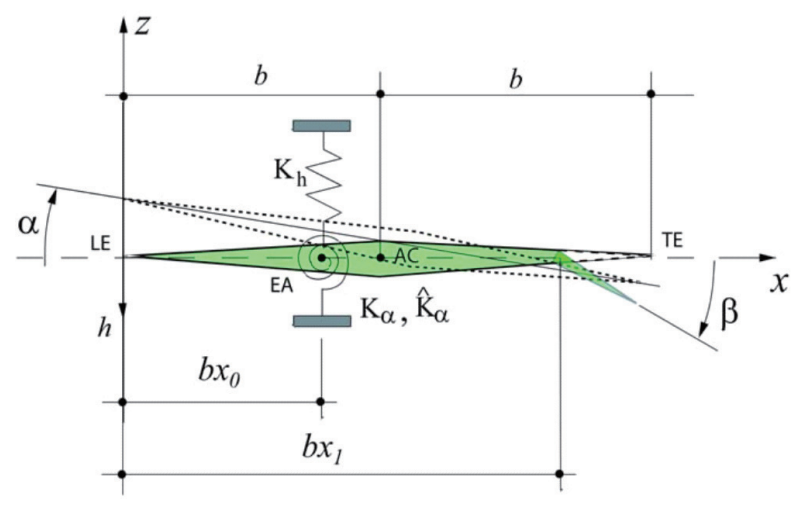

Fig 1. Supersonic wing section with flap to account for the flap deflection is presented next. Within the PTA, the unsteady pressure can be defined as follows

$$
p(y, t)=p_{\infty}\left(1+\frac{\gamma-1}{2} \frac{v_{z}}{a_{\infty}}\right)^{2 \gamma /(\gamma-1)}
$$

where $v_{z}(t)$ and $a_{\infty}$ represent the downwash velocity normal to the airfoil surface and the undisturbed speed of sound respectively, and are defined as follows

$$
v_{z}=\mp\left(w_{t}+U_{\infty} w_{x}\right) ; \quad a_{\infty}^{2}=\gamma p_{\infty} / \rho_{\infty}
$$

In the definition of $v_{z}(t)$, $\mp$ denotes the upper and lower surfaces, respectively, while $U_{\infty}$ denotes the air speed of the undisturbed flow. In the expression (3), $a_{\infty}, p_{\infty}$ and $\rho_{\infty}$ denote the pressure and air density of the undisturbed flow, respectively, while $\gamma$ is the isentropic gas coefficient $(\gamma=$ 1.4 for dry-air). The transverse deflection $w(t)$ in (3) can be expressed as [26]

$$
w(t)=\left\{\begin{array}{cc}
h(t)+\left(x-b x_{0}\right) \alpha(t) & \text { for } x<b x_{1}, \\
h(t)+\left(x-b x_{0}\right) \alpha(t)+\left(x+b x_{1}\right) \beta(t) & \text { for } x \geq b x_{1}
\end{array}\right.
$$

where $x_{0}$ and $x_{1}$ denote the dimensionless location of the elastic axis and of the torsional spring of the flap from the leading edge respectively, while $\beta(t)$ represents the flap displacement. In the binomial expansion of (PTA), the pressure formula for PTA in the third-order approximation can be obtained by retaining the terms up to and including ( $\left.v_{z} / a_{\infty}\right)$ as follows [7], [27]-[29]

$$
\frac{p}{p_{\infty}}=1+\gamma \frac{v_{z}}{a_{\infty}} \lambda+\frac{\gamma(\gamma+1)}{4}\left(\frac{v_{z}}{a_{\infty}} \lambda\right)^{2}+\frac{\gamma(\gamma+1)}{12}\left(\frac{v_{z}}{a_{\infty}} \lambda\right)^{3}
$$

The aerodynamic correction factor, $\lambda=M / \sqrt{M^{2}-1}$, is used to correct the PTA to better approximate the pressure at low supersonic flight speed regime. It is important to note that (2) and (5) are only applicable as long as the transformation through contraction and expansion can be consider isentropic, i.e., as long as the induced show losses are negligible (low-intensity waves). For more details, see [1] [5][30]. PTA provides results in excellent accordance with those based on the Euler solution and the CFL3D code [31]. Considering that flow takes place on both the upper and lower surfaces of the airfoil, $U_{\infty}^{+}=U_{\infty}^{-}=U$; from (3)-(5), the aerodynamic pressure $\delta_{p} \triangleq p-p_{\infty}$ difference can be expressed as

$$
\delta p=-\frac{4 q_{\infty}}{M} \lambda\left[\frac{1}{U_{\infty}} w_{t}+w_{x}+\frac{1+\gamma}{12} \lambda^{2} M^{2}\left(\frac{1}{U_{\infty}} w_{t}+w_{x}\right)^{3}\right](6)
$$

Notice that $\delta_{p}$ also accounts for the deflection of the flap $\beta$. Here, $M=U_{\infty} / \alpha_{\infty}$ is the undisturbed flight Mach number, while $q_{\infty}=\rho_{\infty} U_{\infty}^{2} / 2$ is the undisturbed dynamic pressure as presented in [1] and [3]. The model can be simplified 
to account only for the nonlinearities associated with $\alpha$ and discarding those associated with $\beta$. Even though this is an approximation, the magnitude of the nonlinearities associated with $\beta$ is much smaller than those associated with $\alpha$ and will thus be omitted in this paper. In addition, it is assumed in the following development that the nonlinear aerodynamic damping in (6), i.e., the terms $w_{t}^{3}, w_{t}^{2} w_{x}$, and $w_{t}$ $w_{t}^{2}$ will be discarded and consequently, the cubic nonlinear aerodynamic term reduces to $w_{t}^{3}$ only. Although nonlinear damping can be included in the model, this paper only considers linear damping and thus conservative estimates of the flutter speed are expected.

Finally, the nonlinear aerodynamic lifting and moment can be obtained from the integration of the difference of pressure on the upper and lower surfaces of the airfoil

$$
\begin{aligned}
& L_{a}(t)=\left.\int_{0}^{b x_{1}} \delta p\right|_{x<b x_{1}} d x+\left.\int_{b x_{1}}^{2 b} \delta p\right|_{x \geq b x_{1}} d x \\
& M_{a}(t)=\left.\int_{0}^{b x_{1}} \delta p\right|_{x<b x_{1}}\left(x-b x_{0}\right) d x-\left.\int_{b x_{1}}^{2 b} \delta p\right|_{x \geq b x_{1} x-b x_{0}} d x
\end{aligned}
$$

where $\delta p+_{x<b x_{1}}$ and $\delta p+_{x<b x_{1}}$ are the aerodynamic pressure difference on the clean airfoil and on the flap. In the governing EOM presented in (1), $l_{a}$ and $m_{a}$ denote the counterpart of (7) and (8), which are defined as

$$
\begin{aligned}
l_{a}= & -\frac{\lambda}{12 M \mu}\left\{12 \alpha(t)+M^{2}(1+\gamma) \lambda^{2} \alpha(t)^{3}\right. \\
\left.-3\left[2\left(2-x_{1}\right) \beta(\dot{t})+4\left(-1+x_{0}\right) \dot{\alpha}(t)-\left(-2+x_{1}\right)^{2} \dot{\beta}(t)-4 \dot{\xi}(t)\right]\right\} & \\
m_{a}= & -\frac{\lambda}{12 M \mu r_{\infty}^{2}}\left\{12\left(1-x_{0}\right) \alpha(t)+M^{2}(1+\gamma) \lambda^{2}\left(1-x_{0}\right) \alpha(t)^{3}\right. \\
& -3\left(-2-x_{1}\right)\left(2-2 x_{0}+x_{1}\right) \beta(t)+\left(-2+x_{1}\right)^{2}\left(4-3 x_{0}+x_{1}\right) \\
& \left.+4\left[\left(4-6 x_{0}+3 x_{0}^{2}\right) \dot{\alpha}(t)-3\left(-1+x_{0}\right) \dot{\xi}(t)\right]\right\}
\end{aligned}
$$

Here, $\mu$ represent a the dimensionless mass ratio defined as $m / 4 \rho b^{2}$. Given the definitions above, the governing EOM can be transformed into the following form

$$
\dot{\mathbf{x}}=\mathbf{A} \mathbf{x}+\Phi(y)+\mathbf{G} \beta+\mathbf{G}_{d} \dot{\beta}
$$

where $\mathbf{x}=\left[\begin{array}{llll}\alpha & \dot{\alpha} & \xi & \dot{\xi}\end{array}\right]^{l} \in \mathfrak{R}^{4}$ is a vector of systems states, $\beta(t)$ is a flap deflection control input, while $\mathbf{A}, \mathbf{G}(\mathbf{z})$, $\mathbf{G}_{d}(z)$, and $\boldsymbol{\Phi}(y)$ are defined as follows

$$
\mathbf{A}=\left[\begin{array}{cccc}
0 & 1 & 0 & 0 \\
c_{1} & c_{2} & c_{3} & c_{4} \\
0 & 0 & 0 & 1 \\
k_{1} & k_{2} & k_{3} & k_{4}
\end{array}\right], \Phi(y)=\left[\begin{array}{c}
0 \\
p_{2} \Phi(y) \\
0 \\
p_{4} \Phi(y)
\end{array}\right],
$$

$$
\begin{gathered}
\mathbf{G}=\left[\begin{array}{c}
0 \\
g_{1} \\
0 \\
g_{2}
\end{array}\right], \mathbf{G}_{d}=\left[\begin{array}{c}
0 \\
g_{3} \\
0 \\
g_{4}
\end{array}\right], \\
y=x_{1}, \mathbf{x}=\left[\begin{array}{c}
\alpha \\
\dot{\alpha} \\
\xi \\
\dot{\xi}
\end{array}\right] .
\end{gathered}
$$

where the explicit definitions for the constants $c_{i}, k_{i}, \forall i=$ $1, \ldots, 4$ as well as $p_{2}$ and $p_{4}$ are reported in the Appendix.

\section{Control Objective and Zero Dynamics}

The explicit control objective of this paper is to design a model-free aeroelastic vibration suppression strategy to guarantee the asymptotic convergence of the pitch angle $\alpha$ using the flap deflection $\beta$ as a control input. The secondary objective is to ensure that all system states remain bounded at all times during closed-loop operation. It is assumed that the measurable variables available for control implementation are the pitch angle $\alpha$, pitch angle velocity $\dot{\alpha}$, plunging displacement $\xi$ and plunging displacement velocity $\dot{\xi}$.

Since the proposed control strategy is predicated on the assumption that the system of (12) is minimum phase, the stability of the zero dynamics of the system needs to be assured. For that purpose, the system of (11) is transformed into the following state-space form

$$
\begin{aligned}
& \dot{\eta}=A_{c} \eta+\left[\Phi(y)+B_{c} \bar{\beta}\right] \\
& y=C_{c}^{T} \eta=\left[\begin{array}{llll}
1 & 0 & 0 & 0
\end{array}\right] \eta
\end{aligned}
$$

where $\bar{\beta}=U^{2} \beta$ is an auxiliary control input, $\eta(t)=\left[\eta_{1}(t)\right.$ $\left.\eta_{2}(t) \eta_{3}(t) \eta_{4}(t)\right]^{T} \in \mathfrak{R}^{4}$ is a new vector of system states, while $A_{\mathrm{c}} \in \mathfrak{R}^{4 \times 4}$ and $B_{\mathrm{c}} \in \mathfrak{R}^{4}$ are explicitly defined as follows

$$
A_{c}=\left[\begin{array}{llll}
0 & 1 & 0 & 0 \\
0 & 0 & 1 & 0 \\
0 & 0 & 0 & 1 \\
0 & 0 & 0 & 0
\end{array}\right], \quad B_{c}=\left[\begin{array}{c}
\theta_{1} \\
\theta_{2} \\
\theta_{3} \\
\theta_{4}
\end{array}\right]
$$

where $\theta_{i} \forall_{i}=1,2,3,4$ are constants that are explicitly defined in the Appendix. In (13) above, $\Phi(y) \in \mathfrak{R}^{4}$ denotes a nonlinearity that encodes the nonlinear structural stiffness. It is to be noted here that $\Phi(0)=0$. The state-space system of (13) can be expanded into the following form 


$$
\begin{aligned}
& y=\eta_{1}=\alpha \\
& \dot{\eta}_{1}=\eta_{2}+\Phi_{1}(y)+\theta_{1} \bar{\beta} \\
& \dot{\eta}_{2}=\eta_{3}+\Phi_{2}(y)+\theta_{2} \bar{\beta} \\
& \dot{\eta}_{3}=\eta_{4}+\Phi_{3}(y)+\theta_{3} \bar{\beta} \\
& \dot{\eta}_{4}=\Phi_{4}(y)+\theta_{4} \bar{\beta}
\end{aligned}
$$

Here, the stability of the zero dynamics is studied for the case when the pitch displacement is

regulated to the origin. Mathematically, this implies that

$y \equiv 0 \Rightarrow \dot{y}=\dot{\eta}_{1} \equiv 0$

which implies from the second equation of (15) that

$\bar{\beta}^{*}=-\frac{\eta_{2}}{\theta_{1}}$

Since $\Phi_{1}(0)=0$. The zero dynamics of the system then reduce to the third order system given by

$$
\begin{aligned}
& \dot{\eta}_{2}=\theta_{2} \beta^{*}+\eta_{3} \\
& \dot{\eta}_{3}=\theta_{3} \bar{\beta}^{*}+\eta_{4} \\
& \dot{\eta}_{4}=\theta_{4} \bar{\beta}^{*}
\end{aligned}
$$

Substituting (16) into the above set of equations for $\bar{\beta}^{*}$, we obtain the linear system of equations $\dot{\eta}=A_{c}^{*} \eta$ where $\eta=\left[\eta_{2}\right.$ $\left.\eta_{3} \eta_{4}\right]^{T}$ and $A_{c}^{*}$ is given by

$$
A_{c}^{*}=\left[\begin{array}{ccc}
-\theta_{1}^{-1} \theta_{2} & 1 & 0 \\
-\theta_{1}^{-1} \theta_{3} & 0 & 1 \\
-\theta_{1}^{-1} \theta_{4} & 0 & 0
\end{array}\right]
$$

For the nominal system of (15), the eigenvalues of $A_{c}^{*}$ lie in the left half plane which implies that the zero dynamics of the system are asymptotically stable, i.e., this is a minimum phase system. This implies that asymptotic convergence of the pitching variable $\alpha$ assures the asymptotic convergence of the plunging variable $z$.

\section{Open-Loop Error System Development}

Given the definitions of (13) and (14), $\ddot{\alpha}$ can be expressed as follows

$$
\ddot{\alpha}=c_{1} \alpha+c_{2} \dot{\alpha}+p_{2} \Phi(\alpha)+c_{3} \xi+c_{4} \dot{\xi}+g_{1} \beta+g_{3} \dot{\beta}
$$

The tracking error $e_{1} \triangleq \alpha_{d}-\alpha$ is defined where $\alpha_{d} \in \mathfrak{R}$ denotes the desired output vector which needs to be smooth in deference to the requirements of the subsequent control design. For the control objective, one can simply choose $\alpha_{d}$ to be zero all the time or use another desirable smooth time-varying trajectory $\alpha_{d}(t)$ along which the actual pitching variable $\alpha$ can be driven towards the origin. In order to facilitate the ensuing control design and stability analysis, we also define the tracking error $e_{2}(t) \in \mathfrak{R}$ and the filtered tracking error signal $r(t) \in \mathfrak{R}$ as follows

$$
\begin{aligned}
& e_{2}=\dot{e}_{1}+\lambda_{1} e \\
& r=e_{2}+\lambda_{2} e_{1}
\end{aligned}
$$

where $\lambda_{1}, \lambda_{2}$ are positive constants. By utilizing the definitions above, one can obtain

$$
\dot{r}=\ddot{\alpha}_{d}-\ddot{\alpha}+\lambda_{1} \dot{e}_{1}+\lambda_{2} \dot{e}_{1}
$$

By substituting (17) for $\ddot{\alpha}$ in the above expression, the open-loop dynamics for $r$ can be obtained as follows

$$
\begin{aligned}
\dot{r}=\ddot{\alpha}_{d}-c_{1} \alpha-c_{2} \dot{\alpha}-p_{2} \Phi(\alpha)-c_{3} z & -c_{4} \dot{z}-g_{1} \beta \\
& -g_{3} \dot{\beta}+\lambda_{1} \dot{e}_{1}+\lambda_{2} \dot{e}
\end{aligned}
$$

After a convenient rearrangement of terms, the open-loop dynamics can be rewritten as follows

$$
\begin{aligned}
& \frac{\dot{r}}{\left|g_{3}\right|}=\frac{1}{\left|g_{3}\right|}\left(\ddot{\alpha}_{d}-c_{1} \alpha-c_{2} \dot{\alpha}-p_{2} \Phi(\alpha)\right. \\
& \left.+\lambda_{1} \dot{e}_{1}+\lambda_{2} e_{2}-c_{3} z-c_{4} \dot{z}\right)-\frac{g_{1}}{\left|g_{3}\right|} \beta-\operatorname{sgn}\left(g_{3}\right) \dot{\beta} .
\end{aligned}
$$

In order to design a model-free controller, we define an auxiliary nonlinear signal $N(\cdot)$ as follows

$$
\begin{aligned}
& N\left(\alpha_{d}, \dot{\alpha}_{d}, \ddot{\alpha}_{d}, x_{1}, x_{2}, x_{3}, x_{4}\right) \\
& \triangleq \frac{1}{\left|g_{3}\right|}\left(\ddot{x}_{1 d}-c_{1} \alpha-c_{2} \dot{\alpha}-p_{2} \Phi(\alpha)+\lambda_{1} \dot{e}_{1}+\lambda_{2} e_{2}-c_{3} z-c_{4} \dot{z}\right)
\end{aligned}
$$

By utilizing the definition of (22) above, the open-loop dynamics of the system can be compactly

rewritten as follows

$$
\frac{\dot{r}}{\left|g_{3}\right|}=N\left(x_{1 d}, \dot{x}_{1 d}, \ddot{x}_{1 d}, x_{1}, x_{2}, x_{3}, x_{4}\right)-\frac{g_{1}}{\left|g_{3}\right|} \beta-\operatorname{sgn}\left(g_{3}\right) \dot{\beta}
$$

\section{Control Design and Closed-Loop Error System}

Since the structure of the model is assumed to be unknown in the control design, standard adaptive control cannot be applied. In its lieu, a neural network feedforward compensator $\hat{N}$ along with a robustifying term is proposed to compensate for the function $N$ as defined above in (22). 
By the universal function approximation property [32], the nonlinear function of the system $N$ can be approximated as a three-layer network target function as follows

$$
N\left(x_{1 d}, \dot{x}_{1 d}, \ddot{x}_{1 d}, x_{1}, x_{2}, x_{3}, x_{4}\right)=\mathbf{W}^{T} \sigma\left(\mathbf{V}^{T} \overline{\mathbf{x}}\right)+\varepsilon
$$

as long as $N$ is a general smooth function from $\mathfrak{R}^{7}$ to $\mathfrak{R}^{1}$ and the set of inputs to the function is restricted to a compact set $S$ of $\Re^{7}$. In (24), $\overline{\mathbf{x}}=\left[\begin{array}{llllllll}1 & \alpha_{d} & \dot{\alpha}_{d} & \ddot{\alpha}_{d} & x_{1} & x_{2} & x_{3} & x_{4}\end{array}\right]$

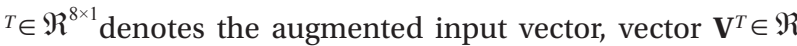
$10 \times 8$ is the ideal first layer interconnection weight matrix between input layer and hidden layer, $\sigma(\cdot) \in \mathfrak{R}^{11 \times 1}$ denotes the sigmoidal activation function, while $\mathbf{W}^{T} \in \mathfrak{R}^{1 \times 11}$ denotes the ideal second layer interconnection weight matrix. In this work, the weight matrixes $\mathbf{W}$ and $\mathbf{V}$ are assumed to be constant and bounded as $\|\mathbf{W}\|_{F} \leq \mathbf{W}_{B}$ and $\|\mathbf{V}\|_{F} \leq \mathbf{V}_{B}$, where $\mathbf{W}_{B}$ and $\mathbf{V}_{B}$ are positive constants. The approximation error is assumed to be bounded in compact set $\|\varepsilon\|<\varepsilon_{N}$ where $\varepsilon_{N}$ is an unknown positive constant related to the number of nodes in the hidden layer.

After substituting the approximation from (24) into (23), one can rewrite the open-loop dynamics as follows

$$
\frac{\dot{r}}{\left|g_{3}\right|}=\mathbf{W}^{T} \boldsymbol{\sigma}\left(\mathbf{V}^{T} \overline{\mathbf{x}}\right)+\varepsilon-g \beta-\operatorname{sgn}\left(g_{3}\right) \dot{\beta}
$$

where $g=\frac{g_{1}}{\left|g_{3}\right|}$. Motivated by the open-loop dynamics and the ensuing stability analysis, the control law is designed as follows

$$
\dot{\beta}=-\operatorname{sgn}\left(g_{3}\right) \hat{g} \beta+\operatorname{sgn}\left(g_{3}\right)\left[\hat{N}+K_{v} r-v+K_{d} \operatorname{sgn}(r)\right](26)
$$

where $K_{v}, K_{d}>0$ are constant control gains, $\hat{N}$ is a typical three-level neural network compensator for target function $N(\overline{\mathbf{x}})$, defined as follows

$$
\hat{N}(\overline{\mathbf{x}})=\hat{\mathbf{W}}^{T} \boldsymbol{\sigma}\left(\hat{\mathbf{V}}^{T} \overline{\mathbf{x}}\right)
$$

$v$ is a robustifying term which will be defined later while $\hat{g}$ is an adaptive estimate for $g$. The dynamic update law for $\hat{g}$ is designed as follows

$$
\dot{\hat{g}}=\operatorname{proj}\{-\Gamma \beta r\}
$$

where the parameter projection operator $\operatorname{proj}\{\cdot\}$ is designed to bound $\hat{g}$ in a known compact set $\Omega$ such that $\operatorname{sgn}\left(g_{3}\right) \hat{g}(t)$ $\geq \varepsilon>0$ for all time. The projection operator defined here is meaningul because the minimum-phase nature of the system ensures that $\operatorname{sgn}\left(g_{3}\right) g(t)=g_{3}^{-1} g_{1}$ is always positive. In (25), $\hat{\mathbf{W}}$ and $\hat{\mathbf{V}}$ are estimates for the neural network interconnection weight matrices that are dynamically generated as follows

$$
\begin{aligned}
& \dot{\hat{\mathbf{W}}}=\left(\mathbf{F} \hat{\boldsymbol{\sigma}}-\mathbf{F} \hat{\boldsymbol{\sigma}}^{\prime} \hat{\mathbf{V}}^{T} \overline{\mathbf{x}}\right) r^{T}-\kappa \mathbf{F}\|r\| \hat{\mathbf{W}} \\
& \dot{\hat{\mathbf{V}}}=\mathbf{G} \overline{\mathbf{x}}\left(\hat{\boldsymbol{\sigma}}^{T} \hat{\mathbf{W}} r\right)^{T}-\kappa \mathbf{G}\|r\| \hat{\mathbf{V}}
\end{aligned}
$$

where $\quad \hat{\boldsymbol{\sigma}} \triangleq \sigma\left(\hat{\mathbf{V}}^{T} \overline{\mathbf{x}}\right), \quad \hat{\boldsymbol{\sigma}}^{\prime} \triangleq d \sigma\left(\hat{\mathbf{V}}^{T} \overline{\mathbf{x}}\right) / d\left(\hat{\mathbf{V}}^{T} \overline{\mathbf{x}}\right), \quad \mathbf{F} \in \mathfrak{R}^{11 \times 11}$ and $G \in \mathfrak{R}^{8 \times 8}$ are positive definite diagonal gain matrixes, while $k>0$ is a scalar design parameter. By substituting the expression for the control law in (26) into the open-loop dynamics of (25) and conveniently rearranging the terms, one can obtain the closed-loop system dynamics as follows

$$
\frac{\dot{r}}{\left|g_{3}\right|}=-K_{v} r+N-\hat{N}+v-K_{d} \operatorname{sgn}(r)+\tilde{g} \beta
$$

where $\tilde{g} \triangleq \hat{g}-g$ is a parameter estimation error. Also note that we can write

$$
\mathbf{W}^{T} \boldsymbol{\sigma}\left(\mathbf{V}^{T} \overline{\mathbf{x}}\right)+\varepsilon-\hat{\mathbf{W}}^{T} \boldsymbol{\sigma}\left(\hat{\mathbf{V}}^{T} \overline{\mathbf{x}}\right)=\tilde{\mathbf{W}}^{T}\left[\hat{\boldsymbol{\sigma}}-\hat{\boldsymbol{\sigma}}^{\prime} \hat{\mathbf{V}}^{T} \overline{\mathbf{x}}\right]+\hat{\mathbf{W}}^{T} \hat{\boldsymbol{\sigma}}^{\prime} \tilde{\mathbf{V}}^{T} \overline{\mathbf{x}}+w
$$

where the weight estimation errors are defined as $\tilde{\mathbf{W}} \triangleq \mathbf{W}$ $-\hat{\mathbf{W}}, \tilde{\mathbf{V}} \triangleq \mathbf{V}-\hat{\mathbf{V}}$ while $w$ is defined as follows

$$
w=\tilde{\mathbf{W}}^{T} \hat{\boldsymbol{\sigma}}^{\prime} \mathbf{V}^{T} \overline{\mathbf{x}}+\tilde{\mathbf{W}}^{T} O\left(\tilde{\mathbf{V}}^{T} \overline{\mathbf{x}}\right)^{2}+\varepsilon(\overline{\mathbf{x}})
$$

To facilitate the subsequent analysis, one can also obtain a compact form representation for $\|\mathbf{w}\|$ as follows

$$
\|w\|=C_{0}+C_{1}\|\tilde{\mathbf{Z}}\|_{F}+C_{2}\|\tilde{\mathbf{Z}}\|_{F}\|r\|
$$

where $C_{0}, C_{1}$ and $C_{2}$ are all positive constants while the ideal composite weight matrix $\mathbf{Z}$, estimated composite weight matrix $\hat{\mathbf{Z}}$, and the composite weight mismatch matrix $\tilde{\mathbf{Z}} \triangleq \mathbf{Z}-\hat{\mathbf{Z}}$ are given as follows

$$
\mathbf{Z}=\left[\begin{array}{cc}
\mathbf{W} & \mathbf{0} \\
\mathbf{0} & \mathbf{V}
\end{array}\right], \quad \hat{\mathbf{Z}} \equiv\left[\begin{array}{cc}
\hat{\mathbf{W}} & \mathbf{0} \\
\mathbf{0} & \hat{\mathbf{V}}
\end{array}\right], \quad \tilde{\mathbf{Z}} \equiv\left[\begin{array}{cc}
\tilde{\mathbf{W}} & \mathbf{0} \\
\mathbf{0} & \tilde{\mathbf{V}}
\end{array}\right]
$$

Per the boundedness property for $\|\mathbf{W}\|_{F}$ and $\|\mathbf{V}\|_{F}$ as described above, there exists a constant $Z_{B}$ such that $Z_{B}>$ $\|\mathbf{Z}\|_{F}$. Based on the definition of $Z_{B}$, the robustifying term $v$ can be designed as

$$
v=-K_{z}\left(\|\hat{\mathbf{Z}}\|_{F}+Z_{B}\right) r
$$

where $K_{z}$ is a positive constant. Finally, it is noted that the functional reconstruction error $\varepsilon(\overline{\mathbf{x}})$ is assumed to be bounded. Thus, the closed-loop dynamics can be finally written as

$$
\frac{\dot{r}}{\left|g_{3}\right|}=-K_{v} r+\tilde{\mathbf{W}}^{T}\left[\hat{\boldsymbol{\sigma}}-\hat{\boldsymbol{\sigma}}^{\prime} \hat{\mathbf{V}}^{T} \overline{\mathbf{x}}\right]+\hat{\mathbf{W}}^{T} \hat{\boldsymbol{\sigma}}^{\prime} \tilde{\mathbf{V}}^{T} \overline{\mathbf{x}}+w+v-K_{d} \operatorname{sgn}(r)+\tilde{g} \beta
$$




\section{Stability Analysis}

In this section, we provide the stability analysis for the proposed model-free controller. We begin by defining a nonnegative Lyapunov function candidate $V_{2}$ as follows

$$
V_{2}=\frac{1}{2\left|g_{3}\right|} r^{2}+\frac{1}{2} \operatorname{tr}\left\{\tilde{W}^{T} F^{-1} \tilde{W}\right\}+\frac{1}{2} \operatorname{tr}\left\{\tilde{V}^{T} G^{-1} \tilde{V}\right\}+\frac{1}{2} \Gamma^{-1} \tilde{g}^{2}
$$

After differentiating $V_{2}$ along the closed-loop dynamics of $\mathrm{r}(\mathrm{t})$ as well as (28), one can obtain the following expression for $\dot{V}_{2}$

$$
\begin{aligned}
& \dot{V}_{2}=r\left[-K_{v} r+\tilde{\mathbf{W}}^{T}\left[\hat{\boldsymbol{\sigma}}-\hat{\boldsymbol{\sigma}}^{\prime} \hat{\mathbf{V}}^{T} \overline{\mathbf{x}}\right]+\hat{\mathbf{W}}^{T} \hat{\boldsymbol{\sigma}}^{\prime} \tilde{\mathbf{V}}^{T} \overline{\mathbf{x}}+w+v(34)\right. \\
& \left.-K_{d} \operatorname{sgn}(r)\right]+\operatorname{tr}\left\{\tilde{\mathbf{W}}^{T} \mathbf{F}^{-1} \dot{\tilde{\mathbf{W}}}\right\}+\operatorname{tr}\left\{\tilde{\mathbf{V}}^{T} \mathbf{G}^{-1} \dot{\tilde{\mathbf{V}}}\right\} \\
& \dot{\hat{\mathbf{W}}}=\left(\mathbf{F} \hat{\boldsymbol{\sigma}}-\mathbf{F} \hat{\boldsymbol{\sigma}}^{\prime} \hat{\mathbf{V}}^{T} \overline{\mathbf{x}}\right) r^{T}-\kappa \mathbf{F}\|r\| \hat{\mathbf{W}} \\
& \dot{\hat{\mathbf{V}}}=\mathbf{G} \overline{\mathbf{x}}\left(\hat{\boldsymbol{\sigma}}^{T} \hat{\mathbf{W}} r\right)^{T}-\kappa \mathbf{G}\|r\| \hat{\mathbf{V}}
\end{aligned}
$$

After applying the neural network weight update laws designed in (29), canceling out the matched terms and utilizing the definitions of (31), (35) can be upperbounded as

$$
\dot{V}_{2} \leq-K_{v} r^{2}+\kappa\|r\| t r\left\{\tilde{\mathbf{Z}}^{T}(\mathbf{Z}-\tilde{\mathbf{Z}})\right\}+\|r\|\|\mathbf{w}\|+r v-r \kappa \operatorname{sgn}(r)
$$

By substituting (30) and (32) into (36), it is possible to further upperbound $\dot{V}_{2}$ as

$$
\begin{aligned}
\dot{V}_{2} \leq & -\|r\|\left[K_{v}\|r\|-\kappa\|\tilde{\mathbf{Z}}\|_{F}\left(Z_{B}-\|\tilde{\mathbf{Z}}\|_{F}\right)-C_{0}-C_{1}\|\tilde{\mathbf{Z}}\|_{F}\right. \\
& \left.-C_{2}\|\tilde{\mathbf{Z}}\|_{F}\|r\|+K_{z}\left(\|\hat{\mathbf{Z}}\|_{F}+Z_{B}\right)\|r\|-K_{d} \operatorname{sgn}(r)\right]
\end{aligned}
$$

where the following relation has been used to derive

$$
\begin{aligned}
\operatorname{tr}\left\{\tilde{\mathbf{Z}}^{T}(\mathbf{Z}-\tilde{\mathbf{Z}})\right\} & =\langle\tilde{\mathbf{Z}}, \mathbf{Z}\rangle-\|\tilde{\mathbf{Z}}\|_{F}^{2} \leq\|\tilde{\mathbf{Z}}\|_{F}\|\mathbf{Z}\|_{F}-\|\tilde{\mathbf{Z}}\|_{F}^{2} \\
& \leq\|\tilde{\mathbf{Z}}\|_{F} Z_{B}-\|\tilde{\mathbf{Z}}\|_{F}^{2} .
\end{aligned}
$$

Based on the fact that $\|\hat{\mathbf{Z}}\|+Z_{B}>\|\tilde{\mathbf{Z}}\|_{F}$, one can choose $K_{z}>$ $C_{2}$ such that (37) can be cast as

$$
\begin{aligned}
& \dot{V}_{2} \leq-\|r\|\left[\mathbf{K}_{v}\|r\|-\kappa\|\tilde{\mathbf{Z}}\|_{F}\left(Z_{B}-\|\tilde{\mathbf{Z}}\|_{F}\right)-C_{0}-C_{1}\|\tilde{\mathbf{Z}}\|_{F}\right] \\
& -r K_{d} \operatorname{sgn}(r) .
\end{aligned}
$$

By defining $C_{3}=Z_{B}+C_{1} / k$ and conveniently rearranging the terms, (40) yields

$$
\begin{aligned}
\dot{V}_{2} \leq-\|r\|\left[\mathbf{K}_{v}\|r\|\right. & \left.+\kappa\left(\|\tilde{\mathbf{Z}}\|_{F}-C_{3} / 2\right)^{2}\right] \\
& +\|r\|\left[C_{0}-\kappa C_{3}^{2} / 4\right]-r k_{d} \operatorname{sgn}(r) .
\end{aligned}
$$

By choosing $K_{d}>\left[C_{0}-k C_{3}^{2} / 4\right]$, one can obtain the following upperbound on $\dot{V}_{2}$

$$
\dot{V}_{2} \leq-\|r\|\left[\mathbf{K}_{v}\|r\|+\kappa\left(\|\tilde{\mathbf{Z}}\|_{F}-C_{3} / 2\right)^{2}\right]
$$

From (34) and (40), it is easy to see that $r \in \mathrm{L}_{2} \cap \mathrm{L}_{\infty}$ while $\hat{g}, \tilde{\mathbf{W}}, \tilde{\mathbf{V}} \in \mathrm{L}_{\infty}$. The boundedness of $r$ implies that $\alpha, \dot{\alpha}$ are bounded by virtue of the definitions of (18) and (19). Since the system is minimum phase and relative degree one, the boundedness of the output guarantees that any first order stable filtering of the input will remain bounded. This implies that all system states remain bounded in closedloop operation which further implies that $N\left(x_{1 d}, \dot{x}_{1 d}, \ddot{x}_{1 d}, x_{1}\right.$, $x_{2}, x_{3}, x_{4}$ ) stays bounded. Since (26) defines a stable filter acting on a bounded input, it is easy to see that $\beta$ and $\dot{\beta}$ stay bounded; furthermore, the flap deflection control input $\beta$ is continuous at all times. The boundedness of $\beta$ implies in turn that $\dot{r} \in \mathrm{L}_{\infty}$ by virtue of the closed-loop dynamics of r. Thus, using previous assertions, one can utilize Barbalat's Lemma [33] to conclude that $r \rightarrow 0$ as $t \rightarrow \infty$ which further implies that $e_{1}, \dot{e}_{1} \rightarrow 0$ as $t \rightarrow \infty$. From the asymptotic stability of the zero dynamics, we can further guarantee that $x_{3}, x_{4} \rightarrow 0$ as $t$ $\rightarrow \infty$. Thus, both the pitching and plunging variables show asymptotic convergence to the origin.

\section{Simulation Results}

In this section, simulation results are presented for an aeroelastic system controlled by the proposed continuous robust controller. The nonlinear aerodynamic model is simulated using the dynamics of (1), (7) and (10).The nominal model parameters are list as follows

Table 1 Controller Parameters

\begin{tabular}{llll}
\hline Parameter & Value & Parameter & Value \\
\hline$K_{z}$ & 0.1 & $Z_{b}$ & 0.1 \\
\hline$\kappa$ & 2 & $K_{v}$ & 100 \\
\hline$K_{d}$ & $1 e-4$ & $\Gamma$ & $1 e-5$ \\
\hline $\mathbf{F}$ & 0.1 & $\mathbf{G}$ & 2 \\
\hline
\end{tabular}




$$
\begin{array}{lll}
\chi_{\alpha}=0.25, & \zeta_{h}=0.01, & x_{0}=0.5, \\
r_{\alpha}=0.5, & \zeta_{\alpha}=0.01, & x_{1}=0.75, \\
b=1.5[\mathrm{~m}], & \gamma=1.4, & \bar{\omega}=1, \\
\lambda=1, & M=2, & V=7.556, \\
\mu=50, & B=5, & \omega_{h}=\omega_{\alpha}=60[\mathrm{~Hz}],
\end{array}
$$

and the controller parameters are listed in Table 1.

The desired trajectory variables $\alpha_{d}, \dot{\alpha}_{d}$ and $\ddot{\alpha}_{d}$ are simply selected as zero. The initial conditions for pitching displacement $\alpha(t)$ and plunging displacement $\xi(t)$ are chosen as $\alpha(0)=5.729 \mathrm{deg}($ about 0.1 radians) and $\xi(0)=0$ $\mathrm{m}$, while all other state variables are initialized to zero. The initial parameter estimate $\hat{g}(0)$ is set to be -1.20 , which is a $10 \%$ shift from its nominal value. The flap deflection $\beta(t)$ is constrained to vary between $\pm 15 \mathrm{deg}$.

The effect of structural nonlinearities on LCO amplitude was analyzed before applying any control. As shown in [22], increase in structural stiffness factor denoted by B led to decrease in LCO amplitude provided the flutter speed

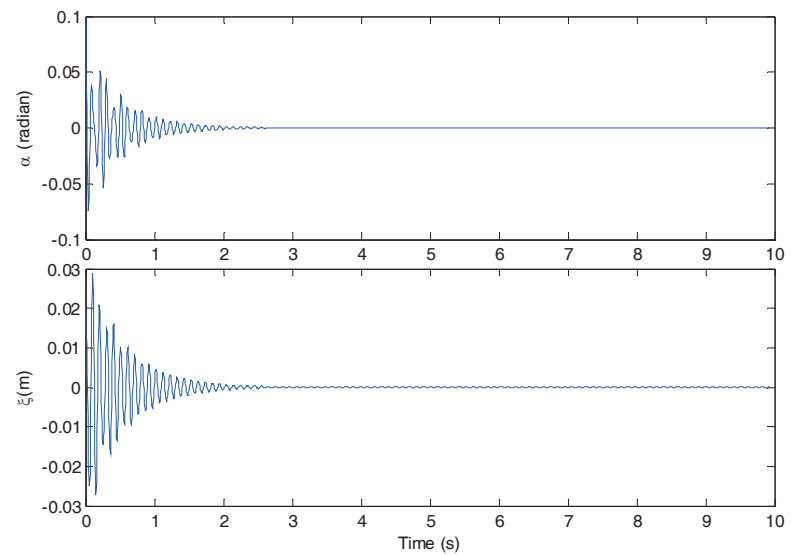

Fig 2. Open-loop dynamics of the aeroelastic system at pre-flutter speed $M=2<M_{\text {futter }}$
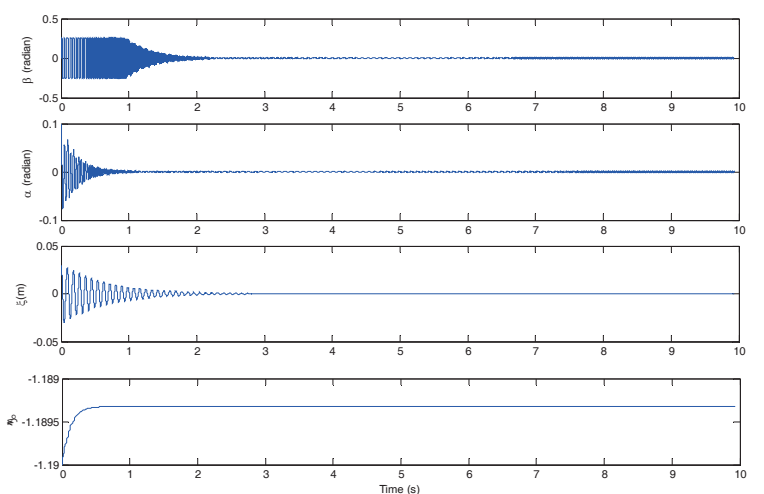

Fig 3. Closed-loop plunging, pitching, control deflection and parameter estimation at pre-flutter speed, $M=2<M_{\text {flutter }}$ remains constant. Furthermore, we also explored the effect of the location of the elastic axis from the leading edge. It was shown in [22] that a decrease in $x_{0}$ leads to decrease in
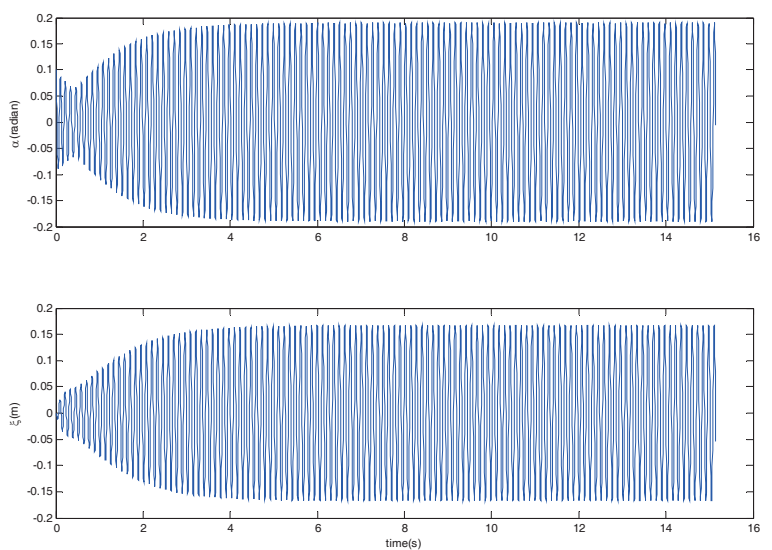

Fig 4. Open-loop dynamics of the aeroelastic system at post-flutter speed $M=3>M_{\text {futter }}$
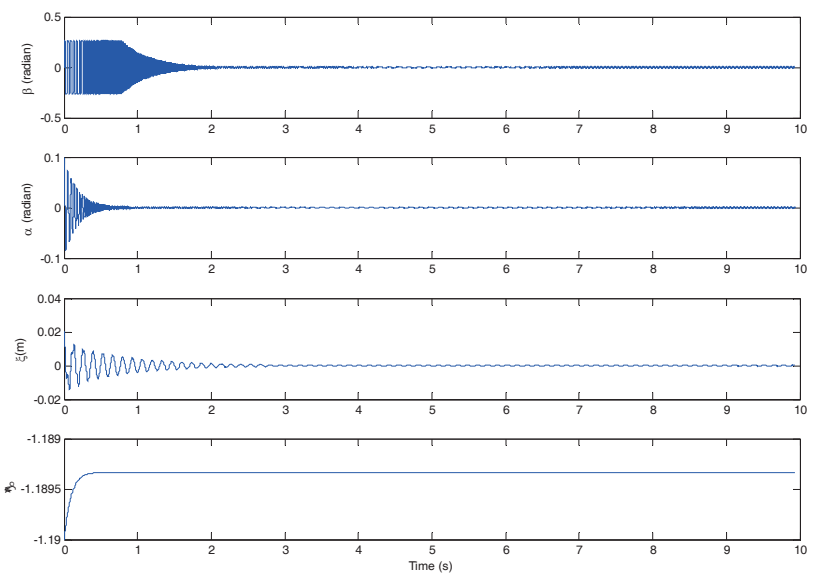

Fig 5. Closed-loop plunging,pitching,control deflections and parameter estimation at post-flutter speed, $M=3>M_{\text {futter }}$
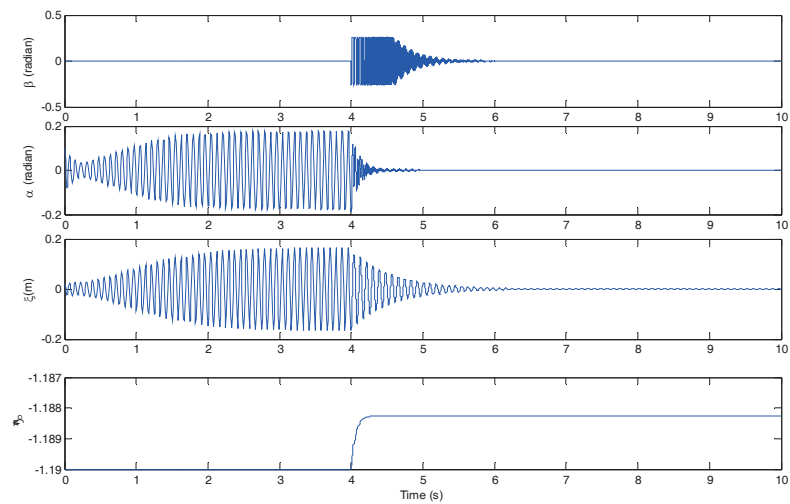

Fig 6. Closed-loop plunging, pitching, control deflection and parameter estimation at post-flutter speed, $M=3>M_{\text {futter; }}$ control applied at $\mathrm{t}=4 \mathrm{~s}$. 
LCO amplitude while the flutter speed increases. It was also shown that increasing the damping ratios $\zeta_{h}$ and $\zeta_{\alpha}$ resulted in decrease of the amplitude of the LCO.

Fig 2 shows the dynamics of open-loop pitching displacement $\alpha$ and plunging displacement $\xi$ at pre-flutter speed. The simulation is carried out in subcritical flight speed regime, $M=2$, below the flutter speed of $M_{\text {flutter }}=2.15$.

Without the controller, it is obvious that the oscillation of pitching degree-of-freedom $\alpha$ will converge within 3[s] while the plunging displacement is lightly damped and it takes over $3[\mathrm{~s}]$ to converge. In Fig 3, it is shown that the proposed robust controller suppresses the oscillation of $\alpha$ in less than 1.5[s] while the plunging displacement $\xi$ is suppressed in $2.5[\mathrm{~s}]$. The parameter estimate $g$ is seen to converge to a constant value within less than $0.5[\mathrm{~s}]$.

Another set of simulations is run for post-flutter speed. As shown in Fig 4, when M is set to be 3, the system dynamics show sustained limit cycle oscillations in open-loop operation. Such LCOs is experienced due to the non-linear pitch stiffness and the aerodynamic nonlinearities. After applying the control to the plant, from Fig 5, it is shown that when the control is turned on at $\mathrm{t}=0[\mathrm{~s}]$, the oscillation of $\alpha$ is suppressed within $1.5[\mathrm{~s}]$. The dynamic oscillatory behavior of the plunging displacement $\xi$ is suppressed within 2.5[s].The control performance is very satisfactory when it start to work at $\mathrm{t}=0$. Next simulation is for delay open of control. In Fig 6, control was turned on at $\mathrm{t}=4[\mathrm{~s}]$ after the system had gone into an LCO. It is seen that the oscillations of the pitching degree $\alpha$ and plunging displacement $\xi$ are suppressed respectively in $1.5[\mathrm{~s}]$ and $3[\mathrm{~s}]$, while in [13] convergence time of the two states are $1.5[\mathrm{~s}]$ and $4[\mathrm{~s}]$. The parameter estimate $\hat{g}$ also converges to a constant in less than $0.5[\mathrm{~s}]$.

These simulation results show that the proposed novel robust controller can effectively suppress the oscillation of both pitching and plunging degrees-of-freedom of the airfoil in both pre-flutter and post-flutter flight speed regimes.

\section{Conclusions}

A modular model-free continuous robust controller was proposed to suppress the aeroelastic vibration characteristics (including flutter and limit cycle oscillations in pre- and post-flutter

condition) of a supersonic 2-DOF lifting surface with flap. Differently from traditional adaptive control strategies, which strictly require the linear parameterization of the system, no prior knowledge of the system model is required for the method presented in this paper. A Lyapunov method based analysis was provided to obtain the global asymptotic stability result. Finally, the simulation results showed that this control strategy can rapidly suppress any aeroelastic vibration

in pre- and post-flutter flight speed regimes.

\section{References}

[1] Librescu, L., Marzocca, P., and Silva, W.A., "Supersonic/ Hypersonic Flutter and Post-flutter of Geometrically Imperfect Circular Cylindrical Panels", Journal of Spacecraft and Rockets, Vol. 39, No. 5, 2002, pp. 802-812.

[2] Mukhopadhyay, V., "Benchmark Active Control Technology”, Journal of Guidance, Control, and Dynamics, Part I 23 (2000) 913-960. Part II 23 (2000) 1093-1139. Part III 24 (2001) 146-192.

[3] Marzocca, P., Librescu L.,Silva W.A., "Flutter, Postflutter and Control of a Supersonic 2-D Lifting Surface”, Journal of Guidance, Control, and Dynamics, vol. 25, no. 5, pp. 962-970, September - October 2002.

[4] Breitbach, E.J., Effects of Structural Non-linearities on Aircraft Vibration and Flutter, AGARD TR-665, North Atlantic Treaty Organization, Neuilly sur Seine, France, 1978.

[5] Librescu, L., "Elastostatics and Kinetics of Anisotropic and Heterogeneous Shell-Type Structures", Aeroelastic Stability of Anisotropic Multilayered Thin Panels, 1st ed., Book series, edited by Leipholz, H., Noordhoff International Publishing, Leyden, The Netherlands, 1975, Chapter 1, pp. 53-63, 106-158, Appendix A, pp. 543-550.

[6] Dowell, E.H., and Ilgamov, M., Studies in non-linear Aeroelasticity, 1st ed., Springer-Verlag, New York, 1988, Chapter 2, pp. 29-63, Chapter 6, pp. 206-277.

[7] Librescu, L., "Aeroelastic Stability of Orthotropic Heterogeneous Thin Panels in the Vicinity of the Flutter Critical Boundary, Part One: Simply supported panels", Journal de Mécanique, Part I, vol. 4, no. 1, pp. 51-76, 1965.

[8] Librescu, L., Aeroelastic Stability of Orthotropic Heterogeneous Thin Panels in the Vicinity of the Flutter Critical Boundary, Part Two Journal de Mécanique, Part II, vol. 6, no. 1, pp. 133-152, 1967.

[9] Pak, C., Friedmann, P.P, and Livne, E., "Digital Adaptive Flutter Suppression and Simulation Using Approximate Transonic Aerodynamics", Journal of Vibration and Control, vol. 1, no. 4, Nov. 1995, pp. 363-388.

[10] Ko., J., Strganac, T.W., and Kurdila, A.J., "Adaptive Feedback Linearization for the Control of a Typical Wing Section with Structural Non-linearity", Non-linear Dynamics, vol. 18 pp. 289-301, 1999.

[11] Xing, W. and Singh, S.N., "Adaptive Output Feedback Control of a Non-linear Aeroelastic Structure", J. Guidance, 
Control and Dynamics, vol. 23, no. 6, pp. 1109-1116, 2000.

[12] Zhang, R. and Singh, S.N., "Adaptive Output Feedback Control of an Aeroelastic System with Unstructured Uncertainties", J. Guidance, Control, and Dynamics, vol. no. 3, pp. 502-509, 2001.

[13] Singh, S.N. and Wang, L., "Output Feedback form and Adaptive Stabilization of a Non-linear Aeroelastic System", J. Guidance, Control and Dynamics, vol. 25, no. 4, pp. 725-732, 2002.

[14] Behal, A., Marzocca, P., Rao V.M., Gnann, A., "Nonlinear adaptive Model Free Control of an Aeroelastic 2-D Lifting Surface", Journal of Guidance, Control and Dynamics, 29(2), pp. 382-390, March-April 2006.

[15] Behal, A., Rao, V. M., Marzocca, P., and Kamaludeen, M., "Adaptive Control for a Nonlinear Wing Section with Multiple Flaps", Journal of Guidance, Control, and Dynamics, vol. 29, no. 3, pp. 744-749, May-June 2006.

[16] Reddy, K.K., Chen, J., Behal, A., and Marzocca, P., "Multi-Input/Multi-Output Adaptive Output Feedback Control Design for Aeroelastic Vibration Suppression", Journal of Guidance, Control, and Dynamics, vol. 30, no. 4, pp. 1040-1048, July-August 2007.

[17] Wang, Z., Behal, A., and Marzocca, P., "Adaptive and Robust Aeroelastic Control of Nonlinear Lifting Surfaces with Single/Multiple Control Surfaces: A Review", International Journal of Aeronautical and Space Science, vol. 11, no. 4, pp. 285-302, December 2010.

[18] Wang, Z., Behal, A., and Marzocca, P., "Robust Adaptive Output Feedback Control Design for a MIMO Aeroelastic System", International Journal of Aeronautical and Space Science, vol. 12, no. 2, pp. 157-167, June 2011.

[19] Zeng, J., Wang, J., de Callafon, R., and Brenner, M., "Suppression of the Aeroelastic/Aeroservoelastic Interaction Using Adaptive Feedback Control Instead of Notching Filters", AIAA Atmospheric Flight Mechanics Conference, Portland, OR, 2011.

[20] Wang, Z., Behal, A., and Marzocca, P., "Continuous Robust Control for Two-Dimensional Airfoils with Leadingand Trailing-Edge Flaps", Journal of Guidance, Control, and Dynamics, vol. 35, no. 2, pp. 510-519, March-April 2012.

[21] Zhang, F. and Söffker, D., "Quadratic Stabilization of a Nonlinear Aeroelastic System Using a Novel Neural-Networkbased controller", Science China Technological Sciences, vol. 54, no. 5, pp. 1126-1133, May 2011.

[22] Wang, Z., Behal, A., Marzocca, P., "Model-Free Control Design for MIMO Aeroelastic System Subject to External Disturbance", J. of Guidance Control and Dynamics, vol. 34, pp. 446-458, 2011.
[23] Rao, V. M., Behal, A., Marzocca, P., Rubillo, C.M., "Adaptive aeroelastic Vibration Suppression of a Supersonic Airfoil with Flap", J. Aerospace Science and Technology, vol. 10, Issue 4, pp. 309--315, May 2006.

[24] Zhang, X., Behal, A., Dawson, D.M., and Xian, B., "Output Feedback Control for a Class of Uncertain MIMO Nonlinear Systems With Non-Symmetric Input Gain Matrix”, in Proc. of IEEE Conference on Decision and Control, Seville, Spain, pp. 7762-7767, 2005.

[25] Chen, J., Behal, A., and Dawson, D.M, "Robust Feedback Control for a Class of Uncertain MIMO Nonlinear Systems", IEEE Transactions on Automatic Control, vol. 53, no. 2, pp. 591-596, Mar. 2008.

[26] Xian, B., Dawson, D. M., "de Queiroz, M. S., and Chen, J., A Continuous Asymptotic Tracking Control Strategy for Uncertain Nonlinear Systems", IEEE Transactions on Automatic Control, vol. 49, no. 7, Jul. 2004, pp. 1206-1211.

[27] Ashley, H. and Zartarian, G., "Piston Theory - A New Aerodynamic Tool for the Aeroelastician", Journal of the Aerospace Sciences, vol. 23, no. 10, 1956, pp. 1109-1118.

[28] Lighthill, M.J., "Oscillating Airfoils at High Mach Numbers", Journal of Aeronautical Science, vol. 20, no. 6, 1953, pp. 402-406.

[29] Liu, D.D., Yao, Z.X., Sarhaddi, D., and Chavez, F.R., "From Piston Theory to a Unified Hypersonic-Supersonic Lifting Surface Method", Journal of Aircraft, vol. 34, no. 3, pp. 304-312, 1997.

[30] Rodden, W.P., Farkas, E.F., Malcom, H.A., and Kliszewski, A.M., Aerodynamic Influence Coefficients from Piston Theory: Analytical Development and Computational Procedure, Aerospace Corporation, TDR-169 (3230-11) TN-2, 1962.

[31] Librescu, L., Chiocchia, G., Marzocca, P., "Implications of Cubic Physical/Aerodynamic Nonlinearities on the Character of the Flutter Instability Boundary", International Journal of Nonlinear Mechanics, 38, pp. 173-199, March 2003.

[32] Thuruthimattam, B.J., Friedmann, P.P., McNamara, J.J., and Powell, K.G., "Aeroelasticity of a Generic Hypersonic Vehicle", $43^{\text {rd }}$ AIAA/ASME/ASCE/AHS/ASC Structures, Structural Dynamics, and Materials Conference, AIAA Paper no. 2002-1209, April 2002.

[33] Hornik, K., Stinchombe, M. and White, H., “Multilayer Feedforward Networks are Universal Approximators", J. Neural Networks, vol. 2, pp. 359-366, March 1989.

[34] Krstic, M., Kanellakopoulos, I., and Kokotovic, P., Nonlinear and Adaptive Control Design, John Wiley \& Sons, New York, 1995. 


\section{Appendix}

The auxiliary constants as well as and that were introduced in the model description of the state-space model are explicitly defined as follows

$$
\begin{gathered}
d=1-\frac{\chi_{\alpha}^{2}}{r_{\alpha}^{2}} \\
k_{1}=\frac{-\lambda}{M \mu d}+\frac{\chi_{\alpha}}{V^{2} d}+\frac{\chi_{\alpha} \lambda\left(1-x_{0}\right)}{M \mu r_{\alpha}^{2} d} ; \\
k_{2}=\frac{-\lambda\left(1-x_{0}\right)}{M \mu d}+\frac{2 \chi_{\alpha} \zeta_{\alpha}}{V d}+\frac{\chi_{\alpha} \lambda\left(4-6 x_{0}+3 x_{0}^{2}\right)}{3 M \mu r_{\alpha}^{2} d} \\
k_{3}=\frac{-\bar{\omega}^{2}}{V^{2} d} ; k_{4}=\frac{-\lambda}{M \mu d}-\frac{2 \zeta_{h} \bar{\omega}}{V d}+\frac{\chi_{\alpha} \lambda\left(1-x_{0}\right)}{M \mu r_{\alpha}^{2} d} \\
c_{2}=\frac{\chi_{\alpha} \lambda\left(1-x_{0}\right)}{M r_{\alpha}^{2} d}-\frac{2 \zeta_{\alpha}}{V d}+\frac{\lambda b\left(4-6 x_{0}+3 x_{0}\right)^{2}}{M \mu r_{\alpha}^{2} d} \\
c_{3}=\frac{\chi_{\alpha} \bar{\omega}^{2}}{r_{\alpha}^{2} V^{2} d} ; c_{4}=\frac{\lambda\left(1-x_{0}\right)}{M \mu r_{\alpha}^{2} d}+\frac{2 \chi_{\alpha} \zeta_{h} \bar{\omega}}{r_{\alpha}^{2} V^{2} d}-\frac{\lambda\left(1-x_{0}\right)}{M \mu r_{\alpha}^{2} d} \\
g_{1}=\frac{\chi_{\alpha} \lambda\left(1-x_{1} / 2\right)}{M \mu r_{\alpha}^{2} d}-\frac{\lambda\left(1-x_{1} / 2\right)\left(1-x_{0}+x_{1} / 2\right)}{M \mu r_{\alpha}^{2} d}
\end{gathered}
$$

$$
\begin{gathered}
g_{2}=\frac{-\lambda\left(1-x_{1} / 2\right)}{M \mu d}+\frac{\chi_{\alpha} \lambda\left(1-x_{1} / 2\right)\left(1-x_{0}+x_{1} / 2\right)}{M \mu r_{\alpha}^{2} d} \\
g_{3}=\frac{\chi_{\alpha} \lambda\left(-1+x_{1} / 2\right)^{2}}{M \mu r_{\alpha}^{2} d}-\frac{\lambda\left(-1+x_{1} / 2\right)^{2}\left(4-3 x_{0}+x_{1}\right)}{3 M \mu r_{\alpha}^{2} d} \\
g_{4}=\frac{-\lambda\left(-1+x_{1} / 2\right)^{2}}{M \mu d}+\frac{\chi_{\alpha} \lambda\left(-1+x_{1} / 2\right)^{2}\left(4-3 x_{0}+x_{1}\right)}{3 M \mu r_{\alpha}^{2} d} \\
p_{2}=\frac{\chi_{\alpha} \lambda^{2} M(1+\gamma)}{12 \mu r_{\alpha}^{2} d}-\frac{\lambda^{2} M(1+\gamma)\left(1-x_{0}\right)}{12 \mu r_{\alpha}^{2} d}+\frac{B}{V^{2} d} \\
p_{4}=-\frac{\lambda^{2} M(1+\gamma)}{12 \mu d}+\frac{\chi_{\alpha} \lambda^{2} M(1+\gamma)\left(1-x_{0}\right)}{12 \mu r_{\alpha}^{2} d}+\frac{\chi_{\alpha} B}{V^{2} d}
\end{gathered}
$$

The elements introduced in (15) are explicitly defined as follows

$$
\begin{gathered}
\theta_{1}=g_{3} \\
\theta_{2}=-c_{3}^{-1}\left[c_{4} k_{3} g_{3}-c_{3} c_{4} g_{4}-c_{3} g_{1}-c_{4} g_{3} k_{3}+c_{3} g_{3} k_{4}\right] \\
\theta_{3}=-c_{3}^{-1}\left[c_{4} k_{3} g_{1}-c_{3} g_{1}-c_{3} c_{4} g_{2}-D g_{4}+c_{5} g_{3} k_{3}-c_{4}^{2} g_{4} k_{3}\right. \\
\left.-c_{4} g_{1} k_{3}-c_{4} k_{3} g_{3} k_{4}+c_{3} c_{4} g_{4} k_{4}+c_{3} g_{1} k_{4}\right] \\
\theta_{4}=-c_{3}^{-1}\left[-D g_{2}+c_{5} g_{1} k_{3}-c_{4}^{2} g_{2} k_{3}-c_{4} k_{3} g_{1} k_{4}+c_{4} c_{3} g_{2} k_{4}\right] \\
D=c_{3}^{2}+c_{3} c_{4} k_{4}-c_{3} c_{4} k_{3} .
\end{gathered}
$$

